\title{
STUDIES OF MICROBIAL POPULATIONS ARTIFICIALLY LOCAL- IZED IN VIVO. II. DIFFERENCE IN ANTITYPHOIDAL ACTIVITIES OF CHLORAMPHENICOL AND CHLORTETRACYCLINE ${ }^{1}$
}

\author{
BY CHARLES A. WERNER 2 AND WALSH MCDERMOTT WITH THE TECHNICAL AS- \\ SISTANCE OF CAROL ADAMS AND REBECKAH DUBOIS
}

\begin{abstract}
(From the Department of Medicine, New York Hospital-Cornell University Medical Center, New York, N.Y.)
\end{abstract}

(Submitted for publication August 5, 1953; accepted January 13, 1954)

It has long been recognized that only a few compounds which exhibit antimicrobial activity in vitro are capable of manifesting comparable activity when introduced into mammalian hosts. The different pharmacologic and microbiologic factors which may be responsible for chemotherapeutic failure have been reviewed elsewhere $(1,2)$. In general, however, if a particular drug is capable of exerting powerful antimicrobial action in vivo against certain pathogens, that drug will be similarly effective against all pathogenic bacteria which are inhibited by low concentrations of the drug in vitro. Infections which have been notable exceptions to this generalization include typhoid fever, brucellosis, and tuberculosis. Although the causative agents have been inhibited in vitro by low concentrations of a number of drugs of proved value in other infections, only a few compounds have been significantly effective against these diseases in humans or experimental animals. Streptomycin, for example, has inhibited Salmonella typhosa in vitro in concentrations comparable on a weight basis to those of chloramphenicol, yet the former drug has been notably ineffective in the treatment of typhoid fever (3). Similar results have been noted for streptomycin in brucellosis ( 3 ) and for subtilin in experimental infections with Mycobacterium $t u$ berculosis (4).

A more recent example of pronounced disparity of drug action in vitro and in vivo has been the virtual ineffectiveness of chlortetracycline in the

\footnotetext{
1 This study was aided in part by grants from Chas. Pfizer \& Co., Brooklyn, N. Y.; the Lederle Laboratories Division, American Cyanamid Co., Pearl River, N. Y.; and the Division of Research Grants and Fellowships, National Institutes of Health, U. S. Public Health Service.

2 Postdoctorate Research Fellow, National Institutes of Health, U. S. Public Health Service during the period in which this study was conducted.
}

treatment of typhoid fever in humans. Both chlortetracycline and chloramphenicol have been demonstrated to be highly active in relatively low concentration against a wide spectrum of pathogenic microorganisms including $S$. typhosa $(5,6)$, and both drugs have been found to be effective in the treatment of a large number of infections including intracellular infections other than typhoid, such as scrub and murine typhus and acute brucellosis (7-9). While chloramphenicol has been highly successful in the treatment of typhoid, chlortetracycline has been only negligibly effective in this disease (10).

Possible explanations for this phenomenon have been the following: (a) Conventional doses of chlortetracycline have failed to provide sufficient amounts of the drug or its active principle in the tissue fluids; (b) Chlortetracycline is rendered ineffective in the body because of the chemical or anatomic nature of the lesion produced by $S$. $t y$ phosa; and (c) Typhoid bacilli subsisting in the host are less susceptible to the action of chlortetracycline than typhoid bacilli growing in vitro.

The purpose of the present study was to investigate the suppressive effects of chlortetracycline and chloramphenicol on $S$. typhosa localized within experimental animals by means of small agar discs and to compare the results with the antityphoidal activities of the two compounds measured in vitro.

\section{MATERIALS AND METHODS}

Drug-susceptibility determinations in vitro. Two-fold serial dilutions in infusion broth and in 20 per cent serum broth, respectively, were made of standard solutions of chlortetracycline ${ }^{8}$ and chloramphenicol ${ }^{*}$ having an ini-

${ }^{8}$ Supplied as Aureomycin Hydrochloride by the Lederle Laboratories Division, American Cyanamid Co., Pearl River, N. Y.

4 Supplied as Chloromycetin by Parke, Davis and Co., Detroit, Mich. 
tial concentration of 200 micrograms per $\mathrm{ml}$. in distilled water. To the $0.5 \mathrm{ml}$. contents of each tube in the dilution series was added an equal volume of $10^{8}, 10^{\circ}$, or $10^{\circ}$ dilution of 18-hour culture of $S$. typhosa in infusion broth. The final concentrations of drug in each series of tubes ranged from 1:2 through 1:1024 of the original standard solution. Each series of tubes was incubated 24 hours at $37^{\circ} \mathrm{C}$., and the end point was the concentration of drug in the first tube in which there was no visible bacterial growth. In order to determine whether the drugs had rendered the culture sterile, the contents of each tube showing no visible growth were subcultured in agar and broth, respectively. Bacterial homogeneity and density of the culture were determined by conventional methods in each experiment.

Drug-parasite reactions in vivo. Triple layer discs of 3 per cent unenriched agar were constructed under sterile conditions in which the central layer was completely encased by transparent agar and consisted of approximately $0.3 \mathrm{ml}$. of a $10^{8}$ dilution of 18-hour culture of $S$. typhosa. Preliminary dilutions of culture were made in 0.85 per cent sterile saline, and the final dilution was made in agar at 45 to $50^{\circ} \mathrm{C}$. in a water bath. Additional discs containing 4 or $5 \mathrm{ml}$. of sterile agar were used in assaying drug content of agar in vivo. This method has been described in detail (11).

Mongrel cats were weighed and were anesthetized with intravenous sodium pentobarbital. The abdomen was shaved and cleansed with tincture of iodine and alcohol, and a laparotomy incision was carried out using aseptic technic. Two freshly prepared discs containing typhoid bacilli and 6 or 7 discs of plain agar were inserted into the peritoneal cavity and were placed so that the discs were in close contact with the peritoneal surfaces but were not in apposition to each other. The wound edges were approximated with long silk sutures so that the incision could be reopened to remove the discs and closed again at intervals as desired.

After the discs had remained in vivo for three or four hours and bacterial multiplication within the agar had entered the logarithmic phase, the animals were given single doses of chlortetracycline either intravenously or orally, or single oral doses of chloramphenicol, respectively. Animals receiving drug orally had been fasted for 18 hours previously. Blood specimens were withdrawn under sterile precautions from an exposed femoral artery or by cardiac puncture and uninoculated discs were removed from the peritoneal cavity simultaneously at $1,2,4,6,24$, and 48 hours after administration of drug. Additional specimens were taken 15 minutes after intravenous doses. Microbiologic assay of the blood serum and agar discs for drug content was carried out using a conventional twofold serial dilution technic. Standard solutions of each drug were assayed concomitantly with the unknown specimens. The details and validity of this method and the penetrability of agar by the two drugs have been demonstrated in a previous study (11).

After the necessary specimens had been obtained at six hours, the wounds were sutured and the animals were al- lowed to regain consciousness. Fifty $\mathrm{ml}$. of dextrose solution was given by stomach tube at this time to prevent dehydration. The cats were reanesthetized and new incisions were made to recover the agar discs at 24 and 48 hours. At these times the discs which had been inoculated with typhoid bacilli were also removed and were examined for colony formation in the depths of the agar. If no colonies were visible at 24 hours the discs were reinserted and were examined again at 48 hours. If no colonies were visible at 48 hours the discs were transferred to deep petri dishes containing infusion broth and were incubated in vitro 24 to 48 hours to determine whether sterilization had occurred.

Discs which were removed from the cats and found to contain visible colonies were opened with a sterile spatula and the organisms were subcultured on agar. Identity of the bacteria was confirmed by morphologic study, gram staining and agglutination in S. typhosa anti-serum. All animals were sacrificed after 48 hours. Other discs inoculated with typhoid bacilli were introduced into cats which did not receive antimicrobial drug and were incubated in infusion broth or sterile saline in large petri dishes to serve as controls of the ability of $S$. typhosa to grow in unenriched agar in animals and in vitro.

\section{RESULTS}

\section{Drug-susceptibility of S. typhosa in vitro}

Parallel determinations were made of the minimum inhibitory concentrations of chlortetracycline and chloramphenicol for different sized inocula of S. typhosa in infusion broth and in 20 per cent serum broth, respectively. The results have been shown in Table I. The minimum inhibitory concentrations of chlortetracycline ranged from 0.4 to 12.5 micrograms per $\mathrm{ml}$. while those of chloramphenicol ranged from 0.4 to 6.2 micrograms per $\mathrm{ml}$. These concentrations were proportional to the size of the inoculum, but there was seldom more than a two-fold increase in the quantity of drug required as the inoculum size increased ten-fold. The median minimal inhibitory concentrations of chlortetracycline at 24 hours were two to four times as high as those of chloramphenicol on a weightvolume basis. Inhibition of $S$. typhosa by both compounds was reversible and nonsterilizing as demonstrated by the fact that typhoid bacilli grew readily when subcultured from the tubes which showed no visible bacterial growth in the dilution series.

\section{Drug-susceptibility of $S$. typhosa in vivo}

The relationship between serum concentrations of drug and the suppressive effects on typhoid ba- 
TABLE I

Parallel determinations of susceptibility of S. typhosa to chlortetracycline and chloramphenicol

\begin{tabular}{|c|c|c|c|c|c|c|c|c|c|c|c|c|c|}
\hline \multirow{5}{*}{ DAY } & \multirow{5}{*}{$\begin{array}{l}\text { MmeER OF BACILLI } \\
\text { PER } M \text { is HOUR } \\
\text { CULTURE } \times 10^{8}\end{array}$} & \multicolumn{6}{|c|}{ MINIMAL $\begin{array}{c}\text { INHIBITORY } \\
\text { MICROGRAMS }\end{array}$} & \multicolumn{6}{|c|}{$\begin{array}{l}\text { CONCENTRATION } \\
\text { PER ML }\end{array}$} \\
\hline & & \multicolumn{6}{|c|}{ CHLORTETRACYCLINE } & \multicolumn{6}{|c|}{ CHLORAMPHENICOL } \\
\hline & & \multicolumn{3}{|c|}{ WFUSION BROTH } & \multirow{2}{*}{\multicolumn{2}{|c|}{$\frac{20 \% \text { SERUM }}{\text { MOCULUM }}$}} & \multirow{2}{*}{$\begin{array}{l}\text { BROTH } \\
\text { SIZE }\end{array}$} & \multicolumn{3}{|c|}{ INFUSION BROTH } & \multicolumn{2}{|c|}{ 20X SERUM } & \multirow{2}{*}{ BROTH } \\
\hline & & \multicolumn{2}{|c|}{ INOCULUM } & S12E & & & & \multicolumn{2}{|c|}{ INOCULUM : } & \multirow{2}{*}{$\begin{array}{l}12 E \\
10^{-7} \\
\end{array}$} & \multicolumn{2}{|c|}{ INOCULUM } & \\
\hline & & $10^{-0}$ & $10^{-6}$ & $10^{-7}$ & $10^{-6}$ & $10^{-6}$ & $10^{-7}$ & $10^{-6}$ & $10^{-6}$ & & $10^{-6}$ & $10^{-6}$ & $10^{-7}$ \\
\hline 1 & 470 & 6.2 & 6.2 & 3.1 & & & & 1.6 & 1.6 & 0.8 & & & \\
\hline 2 & 81 & 12.5 & 6.2 & 6.2 & & & & 1.6 & 1.6 & 1.6 & & & \\
\hline 3 & is & 6.2 & 6.2 & 3.1 & 3.1 & 1.6 & 1.6 & 1.6 & 0.8 & 0.8 & 1.6 & 0.8 & 0.8 \\
\hline 4 & 66 & 3.1 & 3.1 & 1.6 & 6.2 & 1.6 & 0.4 & 1.6 & 0.8 & 0.8 & 0.8 & 0.8 & 0.4 \\
\hline 5 & 600 & 6.2 & 3.1 & 0.8 & 6.2 & 3.1 & 0.8 & 6.2 & .1 .6 & 0.8 & 0.8 & 0.8 & 0.4 \\
\hline 6 & 550 & 12.5 & 6.2 & 6.2 & 12.5 & 3.1 & 1.6 & 1.6 & 1.6 & 0.8 & 1.6 & 1.6 & 1.6 \\
\hline 7 & 600 & |2.5 & 12.5 & 12.5 & & & & 1.6 & 1.6 & 1.6 & & & \\
\hline 8 & 630 & 3.1 & 1.6 & 1.6 & & & & 3.1 & 0.8 & 0.8 & & & \\
\hline 9 & 820 & 3.1 & 3.1 & 1.6 & 6.2 & 1.6 & 0.4 & 1.6 & 1.6 & 0.8 & 1.6 & 0.8 & 0.4 \\
\hline 10 & 580 & 3:1 & 3.1 & 1.6 & 6.2 & 3.1 & 1.6 & 1.6 & 1.6 & 0.8 & 1.6 & 0.8 & 0.4 \\
\hline 11 & 0 & & & & 12.5 & 3.1 & 0.8 & & & & 1.6 & 0.8 & 0.4 \\
\hline & & 601 & 3. & 10 & 6.2 & 3.1 & 0.8 & 1.6 & T.6 & 0.8 & 1.6 & 0.8 & 0.2 \\
\hline
\end{tabular}

cilli in intraperitoneal agar discs in ten cats receiving chlortetracycline and chloramphenicol have been shown graphically in Figure 1. The curves of the serum concentrations can be divided into two groups with respect to the duration of antityphoidal effect. Concentrations of either drug of 6 to 100 micrograms per ml. for 24 hours in the serum of the animals completely inhibited the formation of grossly visible colonies of $S$. typhosa in the agar discs for 48 hours. The corresponding concentrations of drug in the agar discs were 2.6 to 20 micrograms per $\mathrm{ml}$. for chlortetracycline and less than 6.2 to 50 micrograms per ml. for chloramphenicol. In order to produce these high serum concentrations, intravenous doses of chlortetracycline of 50 to $75 \mathrm{mg}$. per $\mathrm{Kg}$. or oral doses of chloramphenicol of 100 to $200 \mathrm{mg}$. per $\mathrm{Kg}$. were required.

Although no visible colonies were detected in the discs removed from these cats at 48 hours, 20 to 30 large colonies of typhoid bacilli regularly appeared in each disc after a further period of in- cubation for 24 hours in broth. Equal numbers of large colonies appeared within 12 to 24 hours in control discs which were incubated in cats which had received no antimicrobial drug and in infusion broth in vitro. No colonies were observed in control discs incubated in saline for 48 hours.

Lower concentrations of the two drugs were less effective in suppressing the growth of $S$. typhosa. Thus, serum concentrations of 0.8 to 25 micrograms per ml. of either drug maintained for 24 hours were effective in preventing the appearance of visible colonies at 24 hours, but after 48 hours 20 to 30 large colonies were present in the depths of the agar. The corresponding concentration of chlortetracycline in the agar discs was 0.6 to 5 micrograms, while chloramphenicol could not be measured. These serum and agar concentrations followed doses of chlortetracycline of $100 \mathrm{mg}$. per $\mathrm{Kg}$. orally or 10 to $20 \mathrm{mg}$. per $\mathrm{Kg}$. intravenously and doses of chloramphenicol of 25 to $50 \mathrm{mg}$. per $\mathrm{Kg}$. orally. 

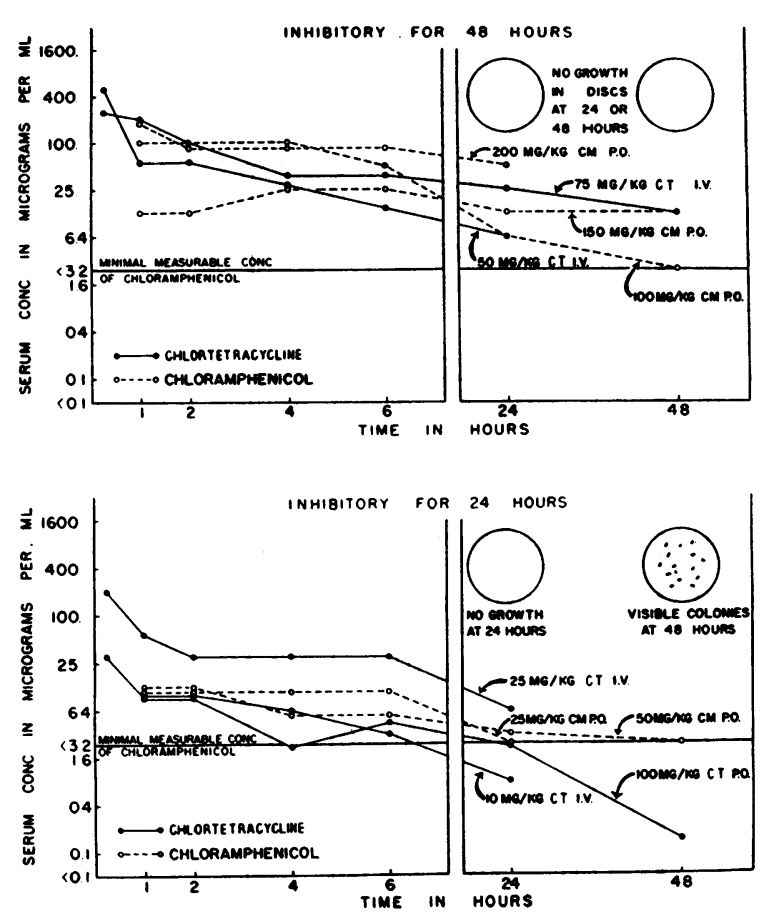

Fig. 1. Effect of Chlortetracycline and ChloramPHENICOL IN SUPPRESSING S. TYPHOSA IN INTRAPERItoneal Agar Discs in Cats

\section{DISCUSSION}

The results of the present investigation indicate that one explanation for the difference of chemotherapeutic effect between chlortetracycline and chloramphenicol in typhoid fever may be a simple difference in the drug-host relationships of the two compounds. Equal serum concentrations of the two drugs exhibited equal effect in suppressing the growth of typhoid bacilli in vivo, but the doses of chlortetracycline which were required to produce these concentrations were much higher than those of chloramphenicol. Intravenous doses of chlortetracycline of 50 and $75 \mathrm{mg}$. per $\mathrm{Kg}$., for example, are equivalent to oral doses of 250 and $375 \mathrm{mg}$. per $\mathrm{Kg}$. and are very much larger than the conventional doses used in clinical practice. In a previous study in humans it was shown that oral doses of chloramphenicol of 50 to $100 \mathrm{mg}$. per $\mathrm{Kg}$. yielded serum concentrations which were well in excess of the minimum inhibitory concentrations of this drug for $S$. typhosa, whereas the same doses of chlortetracycline were inadequate in this respect (12).

The minimum inhibitory concentrations of the two drugs in vitro were not greatly different from each other and were of the same magnitude as those found by other investigators $(5,6)$. Although the inhibitory concentrations of chlortetracycline in the serial dilution method were two to four times greater than those of chloramphenicol on a weightvolume basis, a two-fold difference by this method is within the normal range of error. Moreover, no correction was made for the deterioration of chlortetracycline which may occur at incubator temperatures and which would have the effect of increasing the concentration of this drug required for suppression of bacterial growth (13). Chloramphenicol, on the other hand, has been found to be markedly stable to heat and does not lose potency under these conditions (14). The molar concentrations of the two compounds required to inhibit $S$. typhosa are more nearly equal than the weight-volume concentrations, for the molecular weight of chlortetracycline is almost twice that of chloramphenicol, 508 and 310, respectively (15, 16). Serum concentrations two or more times the minimum inhibitory concentrations in vitro were required for both drugs to prevent the growth of typhoid bacilli in vivo, and when the serum concentrations fell below these values bacterial colonies became visible in the depths of the agar. The bacteriostatic action of the two drugs as opposed to a sterilizing action was confirmed both in vitro and in vivo.

Certain clinical data have also supported the notion that the irregular effect of chlortetracycline in typhoid may be due, at least in part, to a drughost factor and that this may be overcome under certain circumstances. In a study by other investigators $(10)$ two patients with typhoid who received very large doses of chlortetracycline, 350 and $500 \mathrm{mg}$. per $\mathrm{Kg}$., orally, showed a very prompt recovery within 72 hours, and 12 other patients treated with large doses improved more rapidly than untreated controls. In general, however, in the doses ordinarily used in clinical practice chlortetracycline has been relatively ineffective in typhoid fever while chloramphenicol has had a regular and predictable beneficial effect $(10,17)$. This has been in contrast to the high order of therapeutic effectiveness of both drugs in other predominantly intracellular infections such as acute brucellosis and rickettsial infections.

The conditions under which typhoid bacilli exist in agar discs in animals are obviously not the same 
as those in the lesions of typhoid fever. Since the agar was impervious to inflammatory cells of the host in these experiments (11), the bacilli were not situated intracellularly. It was impossible, therefore, by the present method to assay other variables such as intracellular parasitism which conceivably might influence the antityphoidal activity of chlortetracycline and chloramphenicol in vivo. In studies with other microorganisms, however, the tetracycline drugs have been shown to be active in the same concentrations on bacteria situated within monocytes as on bacteria in the extracellular environment $(18,19)$. Whether the other types of host cell parasitized by typhoid bacilli (e.g., plasma cells) (20) are as easily penetrated by the tetracyclines as the monocyte, has not yet been established.

\section{SUMMARY}

A study has been made of the comparative antityphoidal activities of chlortetracycline and chloramphenicol to explain the difference of therapeutic effect of these two compounds in typhoid fever. The in vitro minimum inhibitory concentrations of the two drugs for $S$. typhosa were determined in parallel by the conventional serial dilution method. The in vivo studies were carried out in cats, and the serum concentrations after various oral and intravenous doses of the two drugs were correlated with the suppression of growth of typhoid bacilli encased in triple layered agar discs which were inserted into the peritoneal cavities of the animals. In this way the bacilli were found to be subsisting directly on nutriments supplied by the host in a state more closely approximating true parasitism than is possible in vitro. The agar discs were readily penetrated by both drugs from the extracellular fluid of the peritoneal cavity.

Essentially equivalent concentrations of the two compounds exerted comparable bacteriostatic effect on typhoid bacilli both in vitro and in vivo. The doses of chlortetracycline required to provide concentrations in the extracellular fluid which were inhibitory for $S$. typhosa were found to be significantly greater than those of chloramphenicol and were greater than those doses ordinarily employed in clinical practice. The results of the study indicate, therefore, that one explanation for the difference in the therapeutic activities of chlortetracycline and chloramphenicol in typhoid fever may be the difference in the drug-host, rather than the drug-parasite, relationships of the two compounds. This drug-host difference may be a critical factor in the chemotherapy of typhoid fever in contrast to the treatment of other susceptible intracellular infections in which a more uniform response to these compounds has been noted.

\section{REFERENCES}

1. McDermott, W., Host factors in chemotherapy, in Dubos, R. J., ed., Bacterial and Mycotic Infections of Man, ed. 2, Philadelphia, J. B. Lippincott, 1952, p. 744.

2. Eagle, H., Experimental approach to the problem of treatment failure with penicillin. I. Group A streptococcal infection in mice. Am. J. Med., 1952, 13, 389.

3. Nichols, D. R., and Herrell, W. E., Streptomycin, its clinical uses and limitations. J. A. M. A., 1946, 132, 200.

4. Anderson, H. H., and Wong, S. C., Antibiotics in experimental tuberculosis (with special reference to subtilin). Tuberculology, 1946, 8, 77.

5. Paine, T. F., Jr., Collins, H. S., and Finland, M., Bacteriologic studies on aureomycin. J. Bact., 1948, 56, 489.

6. McLean, I. W., Jr., Schwab, J. L., Hillegas, A. B., and Schlingman, A. S., Susceptibility of microorganisms to chloramphenicol (chloromycetin). J. Clin. Invest., 1949, 28, 953.

7. Knight, V., Ruiz-Sanchez, F., Ruiz-Sanchez, A., and McDermott, W., Aureomycin in typhus and brucellosis. Am. J. Med., 1949, 6, 407.

8. Knight, V., Ruiz-Sanchez, F., and McDermott, W., Chloramphenicol in the treatment of the acute manifestations of brucellosis. Am. J. Med. Sc., 1950, 219, 627.

9. Smadel, J. E., Leon, A. P., Ley, H. L., Jr., and Varela, G., Chloromycetin in the treatment of patients with typhus fever. Proc. Soc. Exper. Biol. \& Med., 1948, 68, 12.

10. Knight, V., Ruis-Sanchez, F., Ruiz-Sanchez, A., Shultz, S., and McDermott, W., Antimicrobial therapy in typhoid. Arch. Int. Med., 1950, 85, 44.

11. Werner, C. A., Knight, V., and McDermott, W., Studies of microbial populations artificially localized in vivo. I. Multiplication of bacteria and distribution of drugs in agar loci. J. Clin. Invest., 1954, 33, 742.

12. Werner, C. A., Knight, V., and McDermott, W. Absorption and excretion of terramycin in humans: comparison with aureomycin and chloramphenicol. Proc. Soc. Exper. Biol. \& Med., 1950, 74, 261.

13. Chandler, C. A., and Bliss, E. A., In vitro studies with aureomycin. Ann. New York Acad. Sc., 1948, $51,221$. 
14. Bartz, Q. R., Isolation and characterization of chloromycetin. J. Biol. Chem., 1948, 172, 445.

15. Broschard, R. W., Dornbush, A. C., Gordon, S., Hutchings, B. L., Kohler, A. R., Krupka, G., Kushner, S., Lefemine, D. V., and Pidacks, C., Aureomycin, a new antibiotic. Science, 1949, 109, 199.

16. Rebstock, M. C., Crooks, H. M., Jr., Controulis, J., and Bartz, Q. R., Chloramphenicol (chloromycetin). IV. Chemical studies. J. Am. Chem. Soc., 1949, 71, 2458.

17. Woodward, T. E., Smadel, J. E., Parker, R. T., and Wissemann, C. L., Jr., Treatment of typhoid fever with antibiotics. Ann. New York Acad. Sc., 1952, 55, 1043.

18. Shaffer, J. M., Kucera, C. J., and Spink, W. W., The protection of intracellular brucella against therapeutic agents and the bactericidal action of serum. J. Exper. Med., 1953, 97, 77.

19. Mackaness, G. B., and Smith, N., The bactericidal action of isoniazid, streptomycin, and terramycin, on extracellular and intracellular tubercle bacilli. Am. Rev. Tuberc., 1953, 67, 322.

20. Goodpasture, E. W., Concerning the pathogenesis of typhoid fever. Am. J. Path., 1937, 13, 175. 